\title{
Variable Transformations for Nearly Singular Integrals in the Boundary Element Method
}

\author{
Dedicated to Professor Masao Iri and Professor Masatake Mori \\ By
}

Ken HAYAMI*

\section{$\S 1$. Introduction}

The Boundary Element Method (BEM) or the Boundary Integral Equation (BIE) method is a convenient method for solving partial differential equations, in that it requires discretization only on the boundary of the domain [2].

In the method, the accurate and efficient computation of boundary integrals is important. In particular, the evaluation of nearly singular integrals, which occur when computing field values near the boundary or treating thin structures, is not an obvious task.

For this purpose, Lachat and Watson [25] proposed an adaptive element subdivision method using an error estimator for the numerical integration. Later, a more sophisticated variable order composite quadrature with exponential convergence was proposed by Schwab [27].

A different approach using quadratic and cubic variable transformations in order to weaken the near singularity before applying Gauss quadrature was introduced by Telles [29]. Koizumi and Utamura [20, 21] used polar coordinates with corrections. Hackbusch and Sauter [7] also used local polar coordinates, performing the inner integrals analytically and the outer integral by Gauss quadrature.

Another approach is to subtract out the near singularity using analytical integration formulas for constant planar elements, and then evaluating the

Communicated by H. Okamoto. Received January 25, 2005. Revised May 25, 2005.

2000 Mathematics Subject Classification(s): 65N38, 65D30, 65D32, 65R20, 41 A55.

Key words: Boundary Element Method, nearly singular integrals, numerical integration, variable transformation, error analysis.

*National Institute of Informatics, 2-1-2, Hitotsubashi, Chiyoda-ku, Tokyo 101-8430, Japan.

e-mail: hayami@nii.ac.jp

(C) 2005 Research Institute for Mathematical Sciences, Kyoto University. All rights reserved. 
remainder term using Gauss quadrature as in Cruse and Aithal [4]. Further, Sládek and Sládek [28] proposed a method to reduce the near singularity of the original boundary integral equation instead of calculating the near singular integral directly.

In this paper, we will review variable transformation methods for evaluating nearly singular integrals over curved surfaces, which were proposed by the author and co-workers [3], [8]-[17], [22]-[24].

The rest of the paper is organized as follows. Section 2 gives a brief explanation of the boundary element formulation of the three-dimensional potential problem. In section 3 , we analyze the nature of integral kernels occurring in such a formulation. In section 4 , we present the outline of the PART method proposed by the author. In section 5 , we treat the radial variable transformation, which is particularly important in the method. In section 6 , we perform an error analysis of the method using complex function theory, which yields insight regarding the optimal radial variable transformation. In section 7 , we mention the use of the double exponential transformation in the radial variable transformation.

\section{§2. Boundary Element Formulation of 3-D Potential Problems}

Let us consider the three-dimensional potential problem as an example. The boundary integral equation is given by

$$
c\left(\boldsymbol{x}_{s}\right) u\left(\boldsymbol{x}_{s}\right)=\int_{\Gamma}\left(q u^{*}-u q^{*}\right) \mathrm{d} \Gamma
$$

where $\boldsymbol{x}_{s}$ is the source point, $u(\boldsymbol{x})$ is the potential, and $q(\boldsymbol{x}):=\frac{\partial u}{\partial n}$ is the derivative of $u$ along the unit outward normal $\boldsymbol{n}$ at $\boldsymbol{x}$ on the boundary $\Gamma$. $\Gamma$ is the boundary of the domain $\Omega$ of interest, and boundary conditions concerning $u$ and $q$ are given on $\Gamma . c\left(\boldsymbol{x}_{s}\right)=1$ when $\boldsymbol{x}_{s} \in \Omega$ and $c\left(\boldsymbol{x}_{s}\right)=\frac{1}{2}$ when $\boldsymbol{x}_{s} \in \Gamma$ and $\Gamma$ is smooth at $\boldsymbol{x}_{s}$.

The fundamental solutions $u^{*}$ and $q^{*}$ are defined by

$$
u^{*}\left(\boldsymbol{x}, \boldsymbol{x}_{s}\right)=\frac{1}{4 \pi r}, \quad q^{*}\left(\boldsymbol{x}, \boldsymbol{x}_{s}\right)=-\frac{(\boldsymbol{r}, \boldsymbol{n})}{4 \pi r^{3}}
$$

where $\boldsymbol{r}:=\boldsymbol{x}-\boldsymbol{x}_{s}$ and $r:=\|\boldsymbol{r}\|_{2}$.

The flux at a point $\boldsymbol{x}_{s} \in \Omega$ is given by

$$
\frac{\partial u}{\partial \boldsymbol{x}_{s}}=\int_{\Gamma}\left(q \frac{\partial u^{*}}{\partial \boldsymbol{x}_{s}}-u \frac{\partial q^{*}}{\partial \boldsymbol{x}_{s}}\right) \mathrm{d} \Gamma
$$


where

$$
\frac{\partial u^{*}}{\partial \boldsymbol{x}_{s}}=\frac{\boldsymbol{r}}{4 \pi r^{3}}, \quad \frac{\partial q^{*}}{\partial \boldsymbol{x}_{s}}=\frac{1}{4 \pi}\left\{\frac{\boldsymbol{n}}{r^{3}}-\frac{3(\boldsymbol{r}, \boldsymbol{n}) \boldsymbol{r}}{r^{5}}\right\} .
$$

Equations (2.1) and (2.3) are discretized on the boundary $\Gamma$ into boundary elements $S_{e}(e=1 \sim N)$ defined by interpolation functions. The integral kernels of equations (2.1) and (2.3) become nearly singular when the distance $d$ between $\boldsymbol{x}_{s}$ and $S_{e}$ is small compared to the size of $S_{e}$. (In the following, we will denote the boundary element $S_{e}$ by $S$ for brevity.)

\section{§3. Nature of Nearly Singular Integral Kernels in 3-D Potential Problems}

First, we will analyze the nature of nearly singular integral kernels occurring in the boundary element formulation of 3-D potential problems. Since near singularity becomes significant in the neighbourhood of the source point $\boldsymbol{x}_{s}$, we will take a planar element $S$ to study the basic nature of the near singular kernels. Let $\overline{\boldsymbol{x}}_{s}$ be the point nearest to $\boldsymbol{x}_{s}$ on $S$. Then, introduce Cartesian coordinates $(x, y, z)$ with $S$ in the $x y$-plane, and polar coordinates $(\rho, \theta)$ in $S$ centred at $\overline{\boldsymbol{x}}_{s}$.

Since $\boldsymbol{x}_{s}=\left(\begin{array}{l}0 \\ 0 \\ d\end{array}\right), \quad \boldsymbol{x}=\left(\begin{array}{l}x \\ y \\ 0\end{array}\right)=\left(\begin{array}{c}\rho \cos \theta \\ \rho \sin \theta \\ 0\end{array}\right), \quad \boldsymbol{r}=\left(\begin{array}{c}\rho \cos \theta \\ \rho \sin \theta \\ -d\end{array}\right), \quad \boldsymbol{n}=\left(\begin{array}{c}0 \\ 0 \\ -1\end{array}\right)$, and $(\boldsymbol{r}, \boldsymbol{n})=d$, equations (2.2) and (2.4) can be expressed as

$$
u^{*}=\frac{1}{4 \pi r}, q^{*}=-\frac{d}{4 \pi r^{3}}, \frac{\partial u^{*}}{\partial \boldsymbol{x}_{s}}=\frac{1}{4 \pi}\left(\begin{array}{c}
\frac{\rho \cos \theta}{r^{3}} \\
\frac{\rho \sin \theta}{r^{3}} \\
-\frac{d}{r^{3}}
\end{array}\right), \frac{\partial q^{*}}{\partial \boldsymbol{x}_{s}}=\frac{1}{4 \pi}\left(\begin{array}{c}
-3 d \frac{\rho \cos \theta}{r^{5}} \\
-3 d \frac{\rho \sin \theta}{r^{5}} \\
-\frac{1}{r^{3}}+\frac{3 d^{2}}{r^{5}}
\end{array}\right) .
$$

For a constant planar element $S$ we have

$$
\int_{S} \mathrm{~d} S=\int_{0}^{2 \pi} \mathrm{d} \theta \int_{0}^{\rho_{\max }(\theta)} \rho \mathrm{d} \rho
$$

using the polar coordinates defined above. Hence, the nearly singular integrals in three-dimensional potential problems involving kernels $u^{*}, q^{*}, \frac{\partial u^{*}}{\partial \boldsymbol{x}_{s}}, \frac{\partial q^{*}}{\partial \boldsymbol{x}_{s}}$ are given in the form

$$
\int_{0}^{2 \pi} \mathrm{d} \theta \int_{0}^{\rho_{\max }(\theta)} \frac{\rho^{\delta}}{r^{\alpha}} \mathrm{d} \rho
$$


Here

$$
I_{\alpha, \delta}:=\int_{0}^{\rho_{j}} \frac{\rho^{\delta}}{r^{\alpha}} \mathrm{d} \rho
$$

where $r=r^{\prime}:=\sqrt{\rho^{2}+d^{2}}$ for planar elements, and $\rho_{j}=1$, for example, can be considered as a model radial variable integral which depicts the essential nature of the nearly singular integrals arising from equations (2.1) and (2.3). The potential integral of equation (2.1) gives rise to $\alpha=\delta=1$ and $\alpha=3, \delta=1$, whereas the flux integral of equation (2.3) gives rise to $\alpha=3, \delta=1,2$ and $\alpha=5, \delta=1,2$.

\section{§4. The Projection and Angular \& Radial Transformation (PART) Method}

As seen in the previous section, nearly singular integrals arising in the three-dimensional boundary element method may be expressed as

$$
I=\int_{S} \frac{f}{r^{\alpha}} \mathrm{d} S
$$

where $S$ is generally a curved surface patch, $r=\left\|\boldsymbol{x}-\boldsymbol{x}_{s}\right\|_{2}$ is the distance between a fixed source point $\boldsymbol{x}_{s}$ and a point $\boldsymbol{x}$ on $S$. $\alpha$ is a positive integer and $f$ is a function of $\boldsymbol{x} \in S$, which does not have any near singularity in $r$. The near singularity of the integrand arises from the denominator $r^{\alpha}$, when the distance between $\boldsymbol{x}_{s}$ and $S$ is small compared to the size of $S$, since the value of the integrand may vary rapidly along $S$ near $\boldsymbol{x}_{s}$.

When $S$ is planar, the integral may have a closed form for some $f$, but this is not the case when $S$ is curved.

The present method was motivated by Telles' method [29], which uses product type Gauss quadrature after applying cubic variable transformations in each of the two variables describing $S$ in order to weaken the near singularity. Let the source distance $d$ be the distance between the source point $\boldsymbol{x}_{s}$ and $S$. It was found that Telles' method does not give accurate results with a reasonable number of quadrature points when $d$ is less than about $1 \%$ compared to the size of $S$. Another drawback of Telles' method when applied to integrals over surfaces is that it concentrates the quadrature points towards the two lines, parallel to the axes in the parameter space defining the curved element, passing through the point corresponding to the source projection, since the method uses the product rule in Cartesian coordinates. 
Our method is based on the observation that, since the near singularity depends on the distance $\left\|\boldsymbol{x}-\boldsymbol{x}_{s}\right\|_{2}$, one should introduce some kind of polar coordinates near $\boldsymbol{x}_{s}$, and then introduce variable transformation along the radial variable, in order to efficiently weaken the near singularity.

Let a point on the curved element $S$ be described by $\boldsymbol{x}\left(\eta_{1}, \eta_{2}\right)$. The method consists of the following steps.

1. Find the point $\boldsymbol{x}\left(\bar{\eta}_{1}, \bar{\eta}_{2}\right)$ on $S$ nearest to $\boldsymbol{x}_{s}$, using Newton-Raphson's method. Compute the source distance $d:=\left\|\boldsymbol{x}_{s}-\boldsymbol{x}\left(\bar{\eta}_{1}, \bar{\eta}_{2}\right)\right\|_{2}$.

2. Determine the point $\tilde{\boldsymbol{x}}_{s}=\tilde{\boldsymbol{x}}\left(\bar{\eta}_{1}, \bar{\eta}_{2}\right)=\sum_{j} \tilde{\phi}_{j}\left(\bar{\eta}_{1}, \bar{\eta}_{2}\right) \boldsymbol{x}_{j}$ on the element $\tilde{S}$ which is obtained by connecting the neighbouring corner nodes $\boldsymbol{x}_{j}$ of the original curved element $S$ by straight lines.

3. Linearly map each sub-triangle $\triangle_{j}$ in the parameter space $\left(\eta_{1}, \eta_{2}\right)$, onto the corresponding sub-triangle $\tilde{\triangle}_{j}: \tilde{\boldsymbol{x}}_{s} \boldsymbol{x}_{j} \boldsymbol{x}_{j+1}$.

4. Introduce polar coordinates $(\rho, \theta)$ centred at $\tilde{\boldsymbol{x}}_{s}$ in each sub-triangle $\tilde{\triangle}_{j}$, to get

$$
I=\sum_{j} \int_{0}^{\triangle \theta_{j}} \mathrm{~d} \theta \int_{0}^{\rho_{j}(\theta)} \frac{f}{r^{\alpha}} J_{j} \rho \mathrm{d} \rho .
$$

Here, $J_{j}$ is the Jacobian of the mapping from Cartesian coordinates on $\tilde{\triangle}_{j}$ to curvilinear coordinates $\left(\eta_{1}, \eta_{2}\right) . \triangle \theta_{j}=\angle \boldsymbol{x}_{j} \tilde{\boldsymbol{x}}_{s} \boldsymbol{x}_{j+1}$.

$$
\rho_{j}(\theta)=\frac{h_{j}}{\cos \left(\theta-\alpha_{j}\right)},
$$

where $h_{j}=\left\|\tilde{\boldsymbol{x}}_{s}-\tilde{\boldsymbol{f}}_{j}\right\|_{2}$ and $\alpha_{j}=\angle \boldsymbol{x}_{j} \tilde{\boldsymbol{x}}_{s} \tilde{\boldsymbol{f}}_{j}$, where $\tilde{\boldsymbol{f}}_{j}$ is the foot of the perpendicular from $\tilde{\boldsymbol{x}}_{s}$ to the edge $\boldsymbol{x}_{j} \boldsymbol{x}_{j+1}$.

5. Transform the radial variable by $R(\rho)$ defined in section 5 in order to weaken the near singularity due to $\frac{1}{r^{\alpha}}$.

6. Transform the angular variable by $t(\theta)$ in order to weaken the near singularity in $\theta$ which arises from $\rho_{j}(\theta)$ when $\tilde{\boldsymbol{x}}_{s}$ is close to the edge of $\tilde{S}$. An efficient transformation can be obtained by letting

$$
\frac{\mathrm{d} \theta}{\mathrm{d} t}=\frac{1}{\rho_{j}(\theta)}
$$

which gives

$$
t(\theta)=\frac{h_{j}}{2} \log \left\{\frac{1+\sin \left(\theta-\alpha_{j}\right)}{1-\sin \left(\theta-\alpha_{j}\right)}\right\} .
$$


7. Apply the product Gauss-Legendre quadrature to perform the numerical integration in the transformed variables $R$ and $t$ in

$$
I=\sum_{j} \int_{t(0)}^{t\left(\triangle \theta_{j}\right)} \frac{\mathrm{d} t}{\rho_{j}(\theta)} \int_{R(0)}^{R\left(\rho_{j}(\theta)\right.} \frac{f J \rho}{r^{\alpha}} \frac{\mathrm{d} \rho}{\mathrm{d} R} \mathrm{~d} R .
$$

Here, we comment on some details of the above procedure.

The Newton-Raphson's method in Step 1 generally converges within 3 to 4 iterations to give a relative error of $10^{-6}$, with the initial solution set to an arbitrary point on $S$, e.g. $\left(\eta_{1}, \eta_{2}\right)=(0,0)$, if $\boldsymbol{x}\left(\bar{\eta}_{1}, \bar{\eta}_{2}\right)$ lies inside the element $S$ $[13,15]$. However, when $\boldsymbol{x}\left(\bar{\eta}_{1}, \bar{\eta}_{2}\right)$ lies outside $S$, the method may diverge. This can be circumvented by constraining the solution on the edge of the element for such cases [14].

It was also found that when the point $\boldsymbol{x}\left(\bar{\eta}_{1}, \bar{\eta}_{2}\right)$ lies outside the original element $S$ in Step 1, or when it lies inside $S$ but very close to the edge of $S$ (namely when $h_{j}<d$ in Steps 3 and 4 ), moving $\boldsymbol{x}\left(\bar{\eta}_{1}, \bar{\eta}_{2}\right)$ to a nearby point on the edge of $S$ and redefining $d$ leads to a considerable reduction of the necessary number of integration points, and hence the computation time [14]-[16].

In Step 2 of the above procedure, the interpolation functions describing the element $\tilde{S}$ are given by $\tilde{\phi}_{j}$, which, in general, is different from the interpolation function $\phi_{j}$ of the original element $S$ [17].

When $S$ is a (curved) quadrilateral element, $\tilde{S}$ is a bilinear quadrilateral element whose vertices coincide with the corner nodes of $S$. (Note that $\boldsymbol{x}_{1}, \boldsymbol{x}_{2}, \boldsymbol{x}_{3}, \boldsymbol{x}_{4}$ and $\tilde{\boldsymbol{x}}_{s}$ are not necessarily coplanar.) The interpolation function defining $\tilde{S}$ is given by

$$
\tilde{\phi}_{k, l}\left(\eta_{1}, \eta_{2}\right)=\tilde{\phi}_{k}\left(\eta_{1}\right) \tilde{\phi}_{l}\left(\eta_{1}\right),
$$

where $k, l=-1,1$ and

$$
\tilde{\phi}_{-1}(\eta)=\frac{1-\eta}{2}, \quad \tilde{\phi}_{1}(\eta)=\frac{\eta+1}{2} .
$$

When $S$ is a (curved) triangular element, $\tilde{S}$ is the planar triangular element whose vertices coincide with the corner nodes of $S$.

Steps 1 and 2 generally consume less than 1\% of the total CPU-time.

In the method, we could also simply work with the sub-triangle $\triangle_{j}$ in the parameter space $\left(\eta_{1}, \eta_{2}\right)$, instead of using the sub-triangle $\tilde{\triangle}_{j}$. However, this gives some problems when the element $S$ has high aspect ratio. Namely, it requires extra integration points for the integration in the angular variable [17] even with the use of an angular variable transformation in the parameter space 
similar to (4.1). This is because the parameter space itself is insensitive to the aspect ratio of the element. Another shortcoming is that the meaning of the source distance $d$ (relative to the element geometry) becomes vague in such cases when one uses it in the radial variable transformation in the parameter space.

We mention here that Koizumi and Utamura [20, 21] also use polar coordinates with further corrections in order to improve accuracy.

The method proposed by Hackbusch and Sauter [7] also employs polar coordinates, but performs the inner integration analytically, while the outer integral is evaluated using the Gauss-Legendre formula. Their method seems promising for planar elements, but theoretical and numerical justification for using it for curved surface elements seems lacking ${ }^{1}$.

\section{$\S 5 . \quad$ Optimal Radial Variable Transformations}

The choice of the variable transformation $R(\rho)$ for the radial variable is particularly important in the PART method.

For constant planar elements,

$$
\rho \mathrm{d} \rho={r^{\prime \alpha}}^{\alpha} R \quad \text { or } \quad R(\rho)=\int \frac{\rho}{r^{\prime \alpha}} \mathrm{d} \rho
$$

where $r^{\prime}:=\sqrt{\rho^{2}+d^{2}}$, is equivalent to performing analytical integration in the radial variable, since $r=r^{\prime}$ in this case.

In [8], we proposed using the above 'singularity cancelling' transformation to curved elements, where $r=r^{\prime}$ does not necessarily hold, in the hope that in the radial variable integration

$$
\int_{R(0)}^{R\left(\rho_{j}(\theta)\right)} \frac{f J \rho}{r^{\alpha}} \frac{\mathrm{d} \rho}{\mathrm{d} R} \mathrm{~d} R=\int_{R(0)}^{R\left(\rho_{j}(\theta)\right)} \frac{f J}{r^{\alpha}} r^{\prime \alpha} \mathrm{d} R
$$

in equation (4.2), the near singularity due to $\frac{1}{r^{\alpha}}$ would be weakened by the term $r^{\prime \alpha}$.

Although this has some effect, it was later found [9] that the $\log \mathrm{L}_{2}$ transformation

$$
\rho \mathrm{d} \rho={r^{\prime}}^{2} \mathrm{~d} R \quad \text { or } \quad R(\rho)=\log \sqrt{\rho^{2}+d^{2}}
$$

\footnotetext{
${ }^{1}$ At p.155 of their paper, it is not explained how to evaluate the second term of $\mathrm{O}\left(h^{\mathrm{min} \cdots}\right)$ in the right hand side of equation (35), which is not generally negligible for curved surface elements.
} 
turns out to be more robust and efficient, in the sense that the transformation works well for all orders of near singularity: $\alpha=1 \sim 5$.

However, this transformation was found to perform poorly for integrals arising in flux calculations as in equation (2.3), or for model radial variable integrals $I_{\alpha, \delta}$ in (3.1) with $\delta=2$. The reason is that the $\log \mathrm{L}_{2}$ transformation of equation (5.1) has the property

$$
\left.\frac{\mathrm{d} \rho}{\mathrm{d} R}\right|_{\rho=+0}=\infty,
$$

so that it induces a infinite derivative at an endpoint of the transformed integrand. This problem can be overcome by the transformation

$$
R(\rho)=\log (\rho+d) \quad\left(\log \mathrm{L}_{1} \text { transformation }\right),
$$

which was shown to work efficiently for flux as well as potential kernels over curved surface elements, and also model integrals (3.1) with $\delta=2$ as well as $\delta=1[11]$.

In [3], parameter tuning by numerical experiments and theoretical error analysis of the transformation

$$
R(\rho)=\log (\rho+a d)
$$

showed that the transformation was optimum around $a=1$, although the transformation is not so sensitive on the parameter $a$.

Another efficient transformation was found to be [16]

$$
R(\rho)=(\rho+d)^{-\frac{1}{5}} \quad\left(\mathrm{~L}_{1}{ }^{-\frac{1}{5}} \text { transformation }\right) .
$$

Tables 1 to 4 give some numerical experiment results comparing the effect of the different transformations. The identity transformation in Table 1 means $R(\rho)=\rho$. Tests were performed on the model radial variable integrals of equation (3.1) where $r=r^{\prime}:=\sqrt{\rho^{2}+d^{2}}$ and $\rho_{j}=1$. The tables give the minimum number of integration points $n$ required for each method to achieve a relative error of $10^{-6}$ for source distance $d$ varying from 10 to $10^{-3}$.

For extensive numerical experiment results on nearly singular integrals over curved surface elements, see [8, 9], [11]-[17]. The results indicate that the proposed method becomes more efficient, in terms of the necessary integration points and CPU-time, compared to previous methods such as Telles' [29] when the source distance $d$ is less than $5 \%$ of the element size.

For planar elements, the method of Hackbusch and Sauter [7] may require less integration points than ours, since the inner integration is done analytically. 
Table 1. Identity Transformation

\begin{tabular}{|r|r||r|r|r|r|r|}
\hline$\alpha$ & \multicolumn{1}{|c||}{$\delta$} & \multicolumn{5}{|c|}{$d$} \\
\cline { 3 - 7 } & & 10 & 1 & $10^{-1}$ & $10^{-2}$ & $10^{-3}$ \\
\hline 1 & 1 & 3 & 5 & 12 & 35 & 80 \\
\hline 3 & 1 & 3 & 6 & 16 & 60 & 190 \\
\hline & 2 & 3 & 5 & 20 & 64 & 210 \\
\hline 5 & 1 & 3 & 6 & 20 & 64 & 210 \\
\hline & 2 & 3 & 7 & 25 & 60 & 190 \\
\hline
\end{tabular}

Table 3. $\log \mathrm{L}_{1}$ Transformation

\begin{tabular}{|r|r||r|r|r|r|r|}
\hline$\alpha$ & \multicolumn{1}{|c||}{$\delta$} & \multicolumn{5}{|c|}{$d$} \\
\cline { 3 - 7 } & & 10 & 1 & $10^{-1}$ & $10^{-2}$ & $10^{-3}$ \\
\hline 1 & 1 & 3 & 5 & 8 & 9 & 8 \\
\hline 3 & 1 & 3 & 5 & 12 & 16 & 20 \\
\hline & 2 & 3 & 6 & 11 & 11 & 16 \\
\hline 5 & 1 & 3 & 6 & 14 & 20 & 25 \\
\hline & 2 & 3 & 6 & 14 & 20 & 20 \\
\hline
\end{tabular}

Table 2. $\log \mathrm{L}_{2}$ Transformation

\begin{tabular}{|r|r||r|r|r|r|r|}
\hline$\alpha$ & \multicolumn{1}{|c||}{$\delta$} & \multicolumn{5}{|c|}{$d$} \\
\cline { 3 - 7 } & & 10 & 1 & $10^{-1}$ & $10^{-2}$ & $10^{-3}$ \\
\hline 1 & 1 & 2 & 3 & 4 & 5 & 6 \\
\hline 3 & 1 & 2 & 3 & 4 & 5 & 6 \\
\hline & 2 & 55 & 55 & 64 & 72 & 80 \\
\hline 5 & 1 & 2 & 3 & 6 & 8 & 10 \\
\hline & 2 & 55 & 64 & 120 & 170 & 200 \\
\hline
\end{tabular}

Table $4 . \mathrm{L}_{1}{ }^{-\frac{1}{5}}$ Transformation

\begin{tabular}{|r|r||r|r|r|r|r|}
\hline$\alpha$ & \multicolumn{1}{|c||}{$\delta$} & \multicolumn{5}{|c|}{$d$} \\
\cline { 3 - 7 } & & 10 & 1 & $10^{-1}$ & $10^{-2}$ & $10^{-3}$ \\
\hline 1 & 1 & 3 & 5 & 7 & 8 & 11 \\
\hline 3 & 1 & 3 & 5 & 9 & 14 & 16 \\
\hline & 2 & 3 & 6 & 10 & 12 & 14 \\
\hline 5 & 1 & 3 & 6 & 11 & 16 & 20 \\
\hline & 2 & 3 & 6 & 12 & 16 & 20 \\
\hline
\end{tabular}

However, their formula includes many terms so that it is not obvious which method is more efficient in terms of CPU-time. For curved surface elements, as mentioned before, the justification for using their method is not clear.

\section{§6. Error Analysis Using Complex Function Theory}

The essential nature of the integration in the radial variable which appear in the 3-D potential problem can be modelled by equation (3.1), which is transformed by $R(\rho)$ as

$$
I=\int_{R(0)}^{R\left(\rho_{j}\right)} \frac{\rho^{\delta}}{r^{\alpha}} \frac{\mathrm{d} \rho}{\mathrm{d} R} \mathrm{~d} R
$$

where $r=\sqrt{\rho^{2}+d^{2}}$. This can be further transformed as

$$
I=\int_{-1}^{1} f(x) \mathrm{d} x
$$

where

$$
f(x):=\frac{\rho^{\delta}}{r^{\alpha}} \frac{\mathrm{d} \rho}{\mathrm{d} R} \frac{\mathrm{d} R}{\mathrm{~d} x}
$$

Here

$$
R:=\frac{\left\{R\left(\rho_{j}\right)-R(0)\right\} x+R\left(\rho_{j}\right)+R(0)}{2} .
$$

The following theorem $[1,30,5]$ gives the error $E_{n}=I-I_{n}$ of the numerical integration $I_{n}=\sum_{j=1}^{n} A_{j} f\left(a_{j}\right)$ of the integral $I=\int_{-1}^{1} f(x) \mathrm{d} x$. 
Theorem 6.1. If $f(z)$ is regular on $K:=[-1,1]$,

$$
E_{n}(f)=\frac{1}{2 \pi \mathrm{i}} \oint_{C} \Phi_{n}(z) f(z) \mathrm{d} z
$$

where

$$
\Phi_{n}=\int_{-1}^{1} \frac{\mathrm{d} x}{z-x}-\sum_{j=1}^{n} \frac{A_{j}}{z-a_{j}}
$$

and the contour $C$ is taken so that it encircles the integration points $a_{1}, a_{2}, \ldots$, $a_{n}$ in the positive (anti-clockwise) direction, and $f(z)$ is regular inside $C$.

The following asymptotic expressions are known for the error characteristic function $\Phi_{n}(z)$ of equation (6.3) for the Gauss-Legendre rule.

1. For $|z| \gg 1[26]$

$$
\Phi_{n}(z)=\frac{c_{n}}{z^{2 n+1}}\left\{1+\mathrm{O}\left(z^{-2}\right)\right\}
$$

where

$$
c_{n}=\frac{2^{2 n+1}(n !)^{4}}{(2 n) !(2 n+1) !}
$$

and $c_{n} \sim \pi 2^{-2 n}$ for $n \gg 1$.

2. For $n \gg 1[1,5]$

- For all $z \in \mathbf{C}$ except for an arbitrary neighbourhood of $K:=[-1,1]$ :

$$
\Phi_{n}(z) \sim 2 \pi\left(z+\sqrt{z^{2}-1}\right)^{-2 n-1}
$$

- For all $z \in \mathbf{C}$ except for an arbitrary neighbourhood of $z=1$ :

$$
\Phi_{n}(z) \sim 2 \mathrm{e}^{-\mathrm{i} \pi} \frac{\mathrm{K}_{0}(2 k \zeta)}{\mathrm{I}_{0}(2 k \zeta)}
$$

where $z=\mathrm{e}^{\mathrm{i} \pi} \cosh (2 \zeta), k=n+\frac{1}{2}$ and $\mathrm{I}_{0}(z), \mathrm{K}_{0}(z)$ are the modified Bessel functions of the first and second kind, respectively.

In the following, let $D:=\frac{d}{\rho_{j}}$, which is the relative source distance. 


\section{§6.1. Error analysis for the $\log \mathrm{L}_{2}$ transformation}

For the $\log \mathrm{L}_{2}$ transformation $R(\rho)=\log \sqrt{\rho^{2}+d^{2}}$ of equation (5.1),

$$
R(0)=\log d, \quad R\left(\rho_{j}\right)=\log r_{j}, \quad r_{j}=\sqrt{\rho_{j}^{2}+d^{2}}
$$

and

$$
\rho(R)=\left(\mathrm{e}^{2 R}-d^{2}\right)^{\frac{1}{2}}
$$

so that

$$
f(z)=a\left\{\mathrm{e}^{\left(-\log \Delta^{\prime}\right) z}-\Delta^{\prime}\right\}^{\frac{\delta-1}{2}} \mathrm{e}^{\frac{2-\alpha}{2}\left(-\log \Delta^{\prime}\right) z}
$$

where

$$
\Delta^{\prime}:=\frac{d}{r_{j}}=\frac{D}{\sqrt{1+D^{2}}}<1, \quad-\log \Delta^{\prime}>0, \quad a:=\frac{\left(-\log \Delta^{\prime}\right)}{2}\left(r_{j} d\right)^{\frac{\delta-\alpha+1}{2}}>0 .
$$

\section{Case: $\delta=$ odd}

Since $\frac{\delta-1}{2}$ is a non-negative integer, $f(z)$ is regular except for $z=\infty$. Hence, taking $C=\{z|| z \mid=R, R \rightarrow \infty\}$ as the contour in Theorem 6.1 and using the asymptotic expression of equation (6.4) for $|z| \gg 1$, we obtain

$$
E_{n}(f)=\frac{c_{n}}{2 \pi \mathrm{i}} \oint_{C} f(z) z^{-2 n-1} \mathrm{~d} z=c_{n} a_{2 n}
$$

where

$$
f(z)=\sum_{k=1}^{\infty} a_{k} z^{k}
$$

so that

$$
E_{n}(f) \sim D^{\frac{\delta+1-\alpha}{2}}\left(\frac{\log D}{n}\right)^{2 n} \sim \mathrm{O}\left(n^{-2 n}\right) .
$$

This corresponds well with numerical results for the integration of potential kernels using the $\log \mathrm{L}_{2}$ transformation [12].

Case: $\delta=$ even

When $\delta$ is even, as in the case of flux kernels, $f(z)$ of equation (6.7) has a branching point singularity at

$$
z_{m}=-1+\mathrm{i} \frac{2 \pi m}{\left(-\log \Delta^{\prime}\right)}, \quad(m: \text { integer }) .
$$


In this case, $f(z)$ has a singularity at the endpoint $z=-1$ of the interval $K=[-1,1]$. However, we can apply Theorem 6.1 by taking the contour as $C=\varepsilon_{\sigma}+l_{+}+C_{\varepsilon}+l_{-}$, where $\varepsilon_{\sigma}$ is an ellipse

$$
\left|z+\sqrt{z^{2}-1}\right|=\sigma, \quad \sigma>1,
$$

with an anti-clockwise direction, which has $z= \pm 1$ as its focii, and the singularities $z_{1}, z_{-1}$ are outside the ellipse. $l_{+}$and $l_{-}$are the real segment $\left(-x_{0},-1-\varepsilon\right)$ in the positive and negative directions, respectively. $x_{0}=\frac{1}{2}\left(\sigma+\frac{1}{\sigma}\right)$ is the major axis of $\varepsilon_{\sigma} . C_{\varepsilon}$ is a circle of radius $0<\varepsilon \ll 1$ in the clockwise direction with its centre at $z=-1$, so that $C$ escapes the singularity at $z=-1$.

It turns out that the most significant contribution to $E_{n}(f)$ of equation (6.2) comes from the branch lines $l_{+}$and $l_{-}$, i.e.,

$$
E_{n}(f) \sim E_{l_{+}, l_{-}} \sim(-\log D)^{\frac{\delta+1}{2}} D^{\delta+1-\alpha} n^{-\delta-1} \sim \mathrm{O}\left(n^{-\delta-1}\right),
$$

where the asymptotic expression $(6.6)$ is used $[12,13]$. This matches well with numerical results for the integration of the flux kernels, which give $E_{n}(f) \sim \mathrm{O}\left(n^{-3}\right)$, where $\delta=2$.

\section{§6.2. Error analysis for the $\log \mathrm{L}_{1}$ transformation}

For the $\log \mathrm{L}_{1}$ transformation $R(\rho)=\log (\rho+d)$, we have

$$
R(0)=\log d, \quad R\left(\rho_{j}\right)=\log \left(\rho_{j}+d\right)
$$

and

$$
\rho(R)=\mathrm{e}^{R}-d, \quad \frac{\mathrm{d} \rho}{\mathrm{d} R}=\mathrm{e}^{R},
$$

so that $f(x)$ of equation (6.1) is given by

$$
f(z)=\frac{b(w-1)^{\delta} w}{\{w-(1-\mathrm{i})\}^{\frac{\alpha}{2}}\{w-(1+\mathrm{i})\}^{\frac{\alpha}{2}}}
$$

where

$$
w:=\mathrm{e}^{\frac{z+1}{2}(-\log \Delta)}, \quad \Delta:=\frac{D}{1+D}<1, \quad-\log \Delta>0, \quad b:=\frac{(-\log \Delta)}{2} d^{\delta-\alpha+1} .
$$

$f(z)$ has singularities (branching when $\alpha=$ odd) at

$$
z=z_{m}^{ \pm}:=-1+\frac{\log 2}{(-\log \Delta)}+\mathrm{i} \frac{\left(4 m \pm \frac{1}{2}\right) \pi}{(-\log \Delta)}, \quad(m: \text { integer }) .
$$


As the contour $C$ in Theorem 6.1, we take the ellipse $\varepsilon_{\sigma}$ of equation (6.8) which passes through the point

$$
z_{t}:=-1+\frac{\log 2}{(-\log \Delta)}+\mathrm{i} \frac{\pi t}{2(-\log \Delta)} \quad(0<t<1),
$$

so that the singularities $z_{0}^{ \pm}$nearest to the endpoint $z=-1$ lie outside $C$. Hence, there are no singularities of $f(z)$ inside $C=\varepsilon_{\sigma}$.

Using the asymptotic expression of equation (6.5) for $n \gg 1$ in equation (6.2), we obtain

$$
\left|E_{n}(f)\right| \leq \frac{l\left(\varepsilon_{\sigma}\right)}{\sigma^{2 n+1}} \max _{z \in \varepsilon_{\sigma}}|f(z)|<2 \pi \sigma^{-2 n} \max _{z \in \varepsilon_{\sigma}}|f(z)|
$$

where $l\left(\varepsilon_{\sigma}\right)$ is the length of the ellipse $\varepsilon_{\sigma}[6]$.

For the ellipse $\varepsilon_{\sigma}$ passing through $z_{t}$, we have

$$
\begin{aligned}
\sigma= & \frac{c}{2} p+\sqrt{\frac{c^{2}}{4} p^{2}-p \log 2+1} \\
& +\sqrt{\frac{c^{2}}{2} p^{2}-p \log 2+\sqrt{\frac{c^{2}}{4} p^{2}-p \log 2+1}}
\end{aligned}
$$

where

$$
p:=\frac{1}{(-\log \Delta)}
$$

and

$$
c:=\sqrt{(\log 2)^{2}+\left(\frac{\pi t}{2}\right)^{2}} \quad(0<t<1) .
$$

$\sigma=\sigma(D, t)$ is a strictly increasing with respect to $D$.

Since $\left|f\left(z_{1}\right)\right|=+\infty$, for $|1-t| \ll 1$, we have

$$
\max _{z \in \varepsilon_{\sigma}}|f(z)| \sim\left|f\left(z_{t}\right)\right| \sim 2^{\frac{\alpha-2}{4}} \pi^{-\frac{\alpha}{2}} d^{\delta-\alpha+1}(-\log \Delta)(1-t)^{-\frac{\alpha}{2}}
$$

from equation (6.9).

Since we are interested in the cases $\alpha=1,3,5,(1-t)^{-\frac{\alpha}{2}} \leq 10$ implies $t \leq 0.6$. Hence, we let $t=0.6$, so that equation (6.12) gives $\sigma=1.31,1.40,1.63$ for the nearly singular cases $D=10^{-3}, 10^{-2}, 10^{-1}$, respectively.

To sum up, for the $\log \mathrm{L}_{1}$ transformation $R(\rho)=\log (\rho+d)$, the numerical integration error is estimated by

$$
E_{n}(f) \sim(-\log D) D^{\delta+1-\alpha} \sigma^{-2 n}
$$


where $\sigma=1.31 \sim 1.63$ for $D=10^{-3} \sim 10^{-1}$. This estimate was found to correspond well with numerical results $[12,13]$.

\section{§6.3. Error analysis for the $\mathrm{L}_{1}^{-\frac{1}{5}}$ transformation}

For the $\mathrm{L}_{1}{ }^{-\frac{1}{m}}$ transformation $R(\rho)=(\rho+d)^{-\frac{1}{m}} \quad(m>1)$, we have

$$
R(0)=d^{-\frac{1}{m}}, \quad R\left(\rho_{j}\right)=\left(\rho_{j}+d\right)^{-\frac{1}{m}}
$$

and

$$
\rho(R)=R^{-m}-d, \quad \frac{\mathrm{d} \rho}{\mathrm{d} R}=-m R^{-m-1},
$$

so that $f(x)$ of equation $(6.1)$ is given by

$$
\begin{aligned}
f(z)=A\{ & \left.\left(z-z_{1}\right)^{m}-\alpha_{1}{ }^{m}\right\}^{\delta}\left(z-z_{1}\right)^{(\alpha-\delta-1) m-1} \\
& \left.\times\left\{\left(z-z_{1}\right)^{m}-2^{-\frac{1}{2}} \mathrm{e}^{\frac{\pi}{4} \mathrm{i}} \alpha_{1}\right\}^{-\frac{\alpha}{2}}\left\{\left(z-z_{1}\right)^{m}-2^{-\frac{1}{2}} \mathrm{e}^{-\frac{\pi}{4} \mathrm{i}} \alpha_{1}\right\}^{m}\right\}^{-\frac{\alpha}{2}}
\end{aligned}
$$

where

$$
\begin{aligned}
& z_{1}:=\frac{1+\Delta^{\frac{1}{m}}}{1-\Delta^{\frac{1}{m}}}, \quad \alpha_{1}:=-\frac{2}{1-\Delta^{\frac{1}{m}}}, \quad \Delta:=\frac{D}{1+D}, \\
& A:=m(-1)^{\delta-m-1} 2^{m-\frac{\alpha}{2}}\left(\rho_{j} D\right)^{\delta-\alpha+1}\left(1-\Delta^{\frac{1}{m}}\right)^{-m}
\end{aligned}
$$

When $m \in \mathbf{N}$, the singularities of $f(z)$ are situated at

$$
z=z_{k}^{ \pm}:=z_{1}+\frac{2^{1-\frac{1}{2 m}}}{1-\Delta^{\frac{1}{m}}} \mathrm{e}^{\left(1 \pm \frac{1}{4 m}+\frac{2 k}{m}\right) \pi \mathrm{i}}
$$

where $k \in \mathbf{Z}$. For $\alpha=\delta=1\left(u^{*}\right)$ and $\alpha=3, \delta=2\left(\frac{\partial u^{*}}{\partial \boldsymbol{x}_{s}}\right), z=z_{1}$ is also a singularity.

As the contour $C$ in Theorem 6.1, again we take the ellipse $\varepsilon_{\sigma}$ of equation (6.8) which does not have any singularities inside. Also, we employ the asymptotic expression of equation (6.5) for $n \gg 1$ in equation (6.2) to obtain equation (6.10).

It can be shown [16] that for $m=5, D>D^{*} \sim 3 \times 10^{-7}$, the ellipse described by equation (6.8) passing through $z=Z_{0}^{-}$is smaller than the one passing through $z=Z_{1}$, and hence the former is the critical one. Hence, for the case $D>D^{*}$, we will consider the ellipse $\varepsilon_{\sigma}$ of equation (6.8) passing through the point: 


$$
z_{t}:=x_{0}+\mathrm{i} t y_{0}=\frac{1+\Delta^{\frac{1}{m}}-2^{1-\frac{1}{2 m}} \cos \frac{\pi}{4 m}}{1-\Delta^{\frac{1}{m}}}+\mathrm{i} \frac{2^{1-\frac{1}{2 m}} \sin \frac{\pi}{4 m}}{1-\Delta^{\frac{1}{m}}} t \quad(0<t<1)
$$

which is located just below the singular point $z_{0}^{-}=x_{0}+\mathrm{i} y_{0}$.

Note that the size $\sigma$ of the ellipse of equation (6.8) passing through a point $z=x+\mathrm{i} y$ is given by $\sigma=\gamma+\sqrt{\gamma^{2}-1}$ where

$$
\gamma:=\frac{\sqrt{(x+1)^{2}+y^{2}}+\sqrt{(x-1)^{2}+y^{2}}}{2} .
$$

Hence, the size of the ellipse of equation (6.8) passing through $z_{t}$ can be determined as a function $\sigma(D, t)$ of $D$ and $t$, where $\sigma(D, t)$ is strictly increasing with respect to $D$.

Since $\left|f\left(z_{1}\right)\right|=+\infty$, for $|1-t| \ll 1$, we have

$$
\begin{aligned}
\max _{z \in \varepsilon_{\sigma}}|f(z)| & \sim\left|f\left(z_{t}\right)\right| \\
& \sim m^{1-\frac{\alpha}{2}} 2^{-\frac{3}{4 \alpha}+\frac{1}{2 m}-\frac{1}{2}}\left(\rho_{j} D\right)^{\delta-\alpha+1}\left(1-\Delta^{\frac{1}{m}}\right)\left(\sin \frac{\pi}{4 m}\right)^{-\frac{\alpha}{2}}(1-t)^{-\frac{\alpha}{2}}
\end{aligned}
$$

from equation (6.13).

Since we are interested in the cases $\alpha=1,3,5,(1-t)^{-\frac{\alpha}{2}} \leq 10$ implies $t \leq 0.6$. Hence, we let $t=0.6$, so that we have $\sigma=1.41,1.48,1.67$ for the nearly singular cases $D=10^{-3}, 10^{-2}, 10^{-1}$, respectively.

To sum up, for the $\mathrm{L}_{1}{ }^{-\frac{1}{5}}$ transformation $R(\rho)=(\rho+d)^{-\frac{1}{5}}$, the numerical integration error is estimated by

$$
E_{n}(f) \sim\left(1-D^{\frac{1}{5}}\right) D^{\delta+1-\alpha} \sigma^{-2 n}
$$

where $\sigma=1.41 \sim 1.67$ for $D=10^{-3} \sim 10^{-1}$, which is slightly better than the corresponding estimate for the $\log \mathrm{L}_{1}$ transformation of equation (6.12). This estimate was also found to correspond well with numerical results $[15,16]$.

\section{§6.4. Error analysis of the identity transformation}

Finally, as a comparison, we analyze the integration error when the identity transformation $R(\rho)=\rho$ is used. In this case,

$$
f(z)=B(z+1)^{\delta}\left(z-z_{1}\right)^{-\frac{\alpha}{2}}\left(z-\bar{z}_{1}\right)^{-\frac{\alpha}{2}}
$$

where

$$
B:=\left(\frac{\rho_{j}}{2}\right)^{\delta+1-\alpha}, \quad z_{1}:=-1+2 D \mathrm{i}
$$


We take the ellipse of equation (6.8) passing through

$$
z_{t}:=-1+2 D t \mathrm{i} \quad(0<t<1)
$$

so that

$$
\sigma=\sqrt{1+(D t)^{2}}+\sqrt{2 D t\left\{\sqrt{1+(D t)^{2}}+D t\right\}}+D t
$$

Since

$$
\max _{z \in \varepsilon_{\sigma}}|f(z)| \sim\left|f\left(z_{t}\right)\right| \sim 2^{-\alpha-1}\left(\rho_{j}\right)^{\delta+1-\alpha} D^{\delta-\frac{3 \alpha}{2}}|1-t|^{-\frac{\alpha}{2}},
$$

letting $t=0.6$ so that $(1-t)^{-\frac{\alpha}{2}} \sim 10$ gives

$$
E_{n}(f) \sim D^{\delta-\frac{3 \alpha}{2}} \sigma^{-2 n}
$$

where $\sigma=1.04,1.12,1.42$ for $D=10^{-3}, 10^{-2}, 10^{-1}$, respectively. These error estimates were also found match well with numerical experiments $[13,15]$.

\section{$\S 6.5$. Summary of the error analysis}

Summing up the error analysis, we have the following.

For the identity transformation $R(\rho)=\rho$,

$$
E_{n}(f) \sim D^{\delta-\frac{3 \alpha}{2}} \sigma^{-2 n}
$$

where $\sigma=1.04,1.12,1.42$ for $D=10^{-3}, 10^{-2}, 10^{-1}$, respectively.

For the $\log \mathrm{L}_{2}$ transformation $R(\rho)=\log \sqrt{\rho^{2}+d^{2}}$,

- when $\delta=$ odd,

$$
E_{n}(f) \sim D^{\frac{\delta+1-\alpha}{2}}\left(\frac{\log D}{n}\right)^{2 n}
$$

- when $\delta=$ even,

$$
E_{n}(f) \sim(-\log D)^{\frac{\delta+1}{2}} D^{\delta+1-\alpha} n^{-\delta-1} .
$$

For the $\log \mathrm{L}_{1}$ transformation $R(\rho)=\log (\rho+d)$,

$$
E_{n}(f) \sim(-\log D) D^{\delta+1-\alpha} \sigma^{-2 n}
$$

where $\sigma=1.31,1.40,1.63$ for $D=10^{-3}, 10^{-2}, 10^{-1}$, respectively. 
For the $\mathrm{L}_{1}{ }^{-\frac{1}{5}}$ transformation $R(\rho)=(\rho+d)^{-\frac{1}{5}}$,

$$
E_{n}(f) \sim\left(1-D^{\frac{1}{5}}\right) D^{\delta+1-\alpha} \sigma^{-2 n}
$$

where $\sigma=1.41,1.48,1.67$ for $D=10^{-3}, 10^{-2}, 10^{-1}$, respectively.

Thus, the $\log \mathrm{L}_{1}$ transformation and the $\mathrm{L}_{1}{ }^{-\frac{1}{5}}$ transformation are predicted to be the most efficient radial variable transformations among the above, where the latter is slightly better than the former.

These error estimates were found to match well with numerical experiments.

The theoretical error estimates also give a clear insight regarding the optimization of the radial variable transformation $R(\rho)$ for nearly singular integrals arising in boundary element analysis.

To be more precise, the singularities $\rho_{ \pm}= \pm d \mathrm{i} \in \mathbf{C}$, inherent in the near singularity of

$$
\frac{1}{r^{\alpha}}=\frac{1}{{\sqrt{\rho^{2}+d^{2}}}^{\alpha}},
$$

are mapped to $R\left(\rho_{ \pm}\right)$by the radial variable transformation $R(\rho)$. Then, $R\left(\rho_{ \pm}\right)$ are mapped to $z_{ \pm}=x\left(R\left(\rho_{ \pm}\right)\right)$by the transformation

$$
x=\frac{2 R-\left\{R\left(\rho_{j}\right)+R(0)\right\}}{R\left(\rho_{j}\right)-R(0)},
$$

in the process of mapping the interval $R:\left[R(0), R\left(\rho_{j}\right)\right]$ to the interval $x:[-1,1]$ in order to apply the Gauss-Legendre rule.

The error analysis in this section showed that the numerical integration error is governed by the maximum size $\sigma$ of the ellipse $\varepsilon_{\sigma}$

$$
\left|z+\sqrt{z^{2}-1}\right|=\sigma, \quad(\sigma>1)
$$

in the complex plane which does not include the singularities $z_{ \pm}$inside.

Therefore, roughly speaking, the optimum radial variable transformation $R(\rho)$ is the transformation which maps the singularities $\rho_{ \pm}= \pm d \mathrm{i}$, inherent in the near singularity, to $z_{ \pm}=x\left\{R\left(\rho_{ \pm}\right)\right\}$which are as far away as possible from the real interval $z:[-1,1]$, allowing an ellipse $\varepsilon_{\sigma}$ of maximum size $\sigma$.

\section{$\S 7 . \quad$ On the Use of the Double Exponential Transformation}

The double exponential (DE) formula [31] is known to be a powerful method for singular integrals and have also been used for nearly singular integrals in the boundary element method $[18,19]$. In $[10,13,15]$, we applied the 
single (SE) and double exponential (DE) formulas to the model radial variable integrals of equation (3.1), in combination with the truncated trapezium rule. However, they were not as efficient as the $\log \mathrm{L}_{1}$ and the $\mathrm{L}_{1}{ }^{-\frac{1}{5}}$ transformations combined with the Gauss-Legendre rule.

Nevertheless, in the context of automatic integration, methods based on the double exponential transformation are attractive. This is because they are based on the trapezium rule with equal step size, so that one can keep on adding integration points, making use of previous integration points, until sufficient accuracy is achieved. In [22]-[24], we showed by theoretical error analysis and numerical experiments on the model radial variable integrals of equation (3.1), that the $\log \mathrm{L}_{2}$ transformation $R(\rho)=\log \sqrt{\rho^{2}+d^{2}}$ in combination with the double exponential transformation gives promising results when using the trapezium rule. These transformations alone, which were not particularly attractive, proved to be useful when combined. This is because the double exponential transformation has the effect of removing the problematic end-point singularity inherent in the $\log \mathrm{L}_{2}$ transformation.

To be more specific, the procedure applied to the model integrals of (3.1) is described as follows.

Step 1: Apply the $\log \mathrm{L}_{2}$ transformation:

$$
R(\rho)=\log \sqrt{\rho^{2}+d^{2}}
$$

and let

$$
x=\frac{2 R-\left\{R\left(\rho_{j}\right)+R(0)\right\}}{R\left(\rho_{j}\right)-R(0)} .
$$

Then, the integrals of (3.1) become

$$
I=\int_{-1}^{1} \frac{\rho^{\delta}}{r^{\alpha}} \frac{\mathrm{d} \rho}{\mathrm{d} R} \frac{\mathrm{d} R}{\mathrm{~d} x} \mathrm{~d} x=\int_{-1}^{1} b\left(a^{x}-\frac{1}{a}\right)^{\frac{\delta-1}{2}} a^{\frac{2-\alpha}{2} x} \mathrm{~d} x \equiv \int_{-1}^{1} g(x) \mathrm{d} x,
$$

where $a=\frac{\sqrt{\rho_{j}^{2}+d^{2}}}{d}, \quad b=\frac{\log a}{2}\left(\sqrt{\rho_{j}^{2}+d^{2}} \cdot d\right)^{\frac{\delta-\alpha+1}{2}}$.

Step 2: Apply the Double Exponential(DE) transformation: $x=\tanh \left(\frac{\pi}{2} \sinh u\right)$. Then,

$$
I=\int_{-\infty}^{\infty} g(x) \frac{\mathrm{d} x}{\mathrm{~d} u} \mathrm{~d} u=\int_{-\infty}^{\infty} f(u) \mathrm{d} u,
$$

where

$$
f(u)=g\left(\tanh \left(\frac{\pi}{2} \sinh u\right)\right) \frac{\frac{\pi}{2} \cosh u}{\cosh ^{2}\left(\frac{\pi}{2} \sinh u\right)}
$$


Step 3: Approximate by the trapezium rule:

$$
I \sim h \sum_{k=-\infty}^{\infty} f(k h),
$$

with an appropriate truncation.

The numerical integration of Step 3 can be done automatically as follows:

Step 3.1: Determine the integration interval $[a, b]$ and the step size $h$ for approximating the integral of equation (7.1), and compute according to the $n$ point formula:

$$
I_{h}=h\left\{\frac{1}{2} f(a)+\sum_{j=1}^{n-2} f(a+j h)+\frac{1}{2} f(b)\right\},
$$

where $h=\frac{b-a}{n-1}$.

Step 3.2: Halve the discretization width $h$ and compute $I_{h / 2}$.

Step 3.3: Determine whether the convergence condition:

$$
\left|\frac{I_{h / 2}-I_{h}}{I_{h / 2}}\right|<\epsilon
$$

is satisfied.

If it is satisfied, end. If it is not satisfied, let $h=h / 2$ and go to Step 3.2.

Table 5 and 6 give numerical experiment results comparing the DE transformation with the $\log \mathrm{L}_{2}$-DE transformation, showing the effectiveness of combining the $\log \mathrm{L}_{2}$ and the DE transformations. Tests were performed on the same model radial variable integrals as in Table 1 to 4 , with the same conditions.

In [24], error estimates were also derived for the above transformations and it was shown that combining the $\log \mathrm{L}_{2}$ transformation with the DE transformation has the effect of increasing the distance between the singularity and the real axis, thus improving the accuracy of the quadrature. 
Table 5. DE Transformation

\begin{tabular}{|r|r||r|r|r|r|r|}
\hline$\alpha$ & \multicolumn{1}{|c||}{$\delta$} & \multicolumn{5}{|c|}{$d$} \\
\cline { 3 - 7 } & & 10 & 1 & $10^{-1}$ & $10^{-2}$ & $10^{-3}$ \\
\hline 1 & 1 & 15 & 18 & 26 & 32 & 34 \\
\hline 3 & 1 & 15 & 19 & 36 & 52 & 70 \\
\hline & 2 & 15 & 18 & 32 & 47 & 63 \\
\hline 5 & 1 & 15 & 19 & 36 & 51 & 67 \\
\hline & 2 & 15 & 20 & 40 & 50 & 68 \\
\hline
\end{tabular}

Table 6. $\log \mathrm{L}_{2}$-DE Transformation

\begin{tabular}{|r|r||r|r|r|r|r|}
\hline$\alpha$ & \multicolumn{1}{|c||}{$\delta$} & \multicolumn{5}{|c|}{$d$} \\
\cline { 3 - 7 } & & 10 & 1 & $10^{-1}$ & $10^{-2}$ & $10^{-3}$ \\
\hline 1 & 1 & 14 & 15 & 18 & 20 & 20 \\
\hline 3 & 1 & 14 & 15 & 18 & 20 & 20 \\
\hline & 2 & 14 & 14 & 16 & 18 & 18 \\
\hline 5 & 1 & 14 & 16 & 19 & 18 & 18 \\
\hline & 2 & 14 & 16 & 22 & 23 & 21 \\
\hline
\end{tabular}

\section{$\S 8 . \quad$ Conclusions}

In this paper we reviewed variable transformation methods for evaluating nearly singular integrals over curved surfaces arising in the three-dimensional boundary element method, which were proposed by the author and co-workers. Particularly, we showed that certain nonlinear radial variable transformations play an important role in the methods, and that error analysis using complex function theory yields a clear insight regarding the optimization of the radial variable transformation.

\section{Acknowledgements}

The author would like to thank the referee for useful comments.

\section{References}

[1] Barret, W., Convergence properties of Gaussian quadrature formulae, Comput. J., 3 (1960), 272-277.

[2] Brebbia, C. A., Telles, J. C. F. and Wrobel, L. C., Boundary Element Techniques: Theory and Applications in Engineering, Springer-Verlag, Berlin, 1984.

[3] Choraku, A., On the Optimization and Theoretical Error Analysis of Variable Transformation-Type Numerical Quadrature for the Boundary Element Method, Bachelor Thesis, Department of Mathematical Engineering and Information Physics, The University of Tokyo, 1996 (in Japanese).

[4] Cruse, T. A. and Aithal, R., Non-singular boundary integral equation implementation, Int. J. Numer. Methods Eng., 36 (1993), 237-254.

[5] Donaldson, J. D. and Elliot, D., A unified approach to quadrature rules with asymptotic estimates of their remainders, SIAM J. Numer. Anal., 9 (1972), 573-602.

[6] Davis, P. J. and Rabinowitz, P., Methods of Numerical Integration, Academic Press, 1984.

[7] Hackbusch, W. and Sauter, S. A., On numerical cubature of nearly singular surface integrals arising in BEM collocation, Computing, 52 (1994), 139-159.

[8] Hayami, K. and Brebbia, C. A., A new coordinate transformation method for singular and nearly singular integrals over general curved boundary elements, in C. A. Brebbia, W. L. Wendland, G. Kuhn (eds.), Boundary Elements IX, Proc. 9th Int. Conf. on Boundary Elements, Stuttgart, 1987, Computational Mechanics Publications with Springer-Verlag, Berlin, 1 (1987), 375-399. 
[9] Hayami, K. and Brebbia, C. A., Quadrature methods for singular and nearly singular integrals in 3-D boundary element method (Invited paper), in C. A. Brebbia (ed.), Boundary Elements X, Proc. 10th Int. Conf. on Boundary Element Methods, Southampton, 1988, Computational Mechanics Publications with Springer-Verlag, Berlin, 1 (1988), 237-264.

[10] Hayami, K., High precision numerical integration methods for 3-D boundary element analysis, IEEE Trans. Magnetics, 26 (1990), 603-606.

[11] - A robust numerical integration method for three-dimensional boundary element analysis, in M. Tanaka, C. A. Brebbia and T. Honma (eds.), Boundary Elements XII, Proc. 12th Int. Conf. on Boundary Elements in Engineering, Sapporo, 1990, Computational Mechanics Publications with Springer-Verlag, Berlin, 1 (1990), 33-51.

[12] - A robust numerical integration method for 3-D boundary element analysis and its error analysis using complex function theory, in T. O. Espelid and A. Genz (eds.), Numerical Integration, Proc. NATO Advanced Research Workshop on Numerical Integration, Bergen, 1991, Kluwer Academic Publishers, (1992), 235-248.

[13] _ A Projection Transformation Method for Nearly Singular Surface Boundary Element Integrals, Thesis Submitted for the Degree of Doctor of Philosophy of the Council for National Academic Awards, Computational Mechanics Institute, Wessex Institute of Technology, Southampton, U.K., 1991. (Also available as C. A. Brebbia and S. A. Orszag (eds.), Lect. Notes Eng., 73, Springer-Verlag, Berlin, 1992.)

[14] Hayami, K., Matsumoto, H. and Moroga, K., Improvement and implementation of PART: Numerical quadrature for nearly singular boundary element integrals, in C. A. Brebbia, J. Dominguez and F. Paris (eds.), Boundary Elements XIV, Proc. 14th Int. Conf. on Boundary Element Methods, Seville, 1992 Computational Mechanics Publications with Elsevier Science Publishers, 1 (1992), 605-617.

[15] Hayami, K., Numerical Quadrature for Nearly Singular Integrals in the Three Dimensional Boundary Element Method, Ph.D. Thesis, The University of Tokyo, 1992.

[16] Hayami, K. and Matsumoto, H., A numerical quadrature for nearly singular boundary element integrals, Eng. Anal. Bound. Elem., 13 (1994), 143-154.

[17] , Improvement of quadrature for nearly singular integrals in 3D-BEM, in C. A. Brebbia ed., Boundary Elements XVI, Proc. 16th Int. Boundary Element Method Conference, (1994), 201-210, Computational Mechanics Publications, Southampton.

[18] Higashimachi, T., Okamoto, N., Ezawa, Y., Aizawa, T. and Ito, A., Interactive structural analysis system using the advanced boundary element method, in C. Brebbia, T. Futagami and M. Tanaka eds., Boundary Elements V, Proc. 5th Int. Conf., Hiroshima, (1983), 847-856, Springer-Verlag, Berlin and CML Publications, Southampton.

[19] Jun, L., Beer, G. and Meek, J. L., The application of double exponential formulas in the boundary element method, in C. Brebbia and G. Maier eds., Boundary Elements VII, Proc. 7th Int. Conf., Como, Italy, Springer-Verlag, 2 (1985), 13.3-13.17.

[20] Koizumi, M. and Utamura, M., A new approach to singular kernel integration for general curved elements, Boundary Elements VIII, Proc. 8th Int. Conf., Tokyo, Springer-Verlag, (1986), 665-675.

[21] , A polar coordinate integration scheme with a hierarchical correction procedure to improve numerical accuracy, in T. A. Cruse ed., Proc. IUTAM Symp. on Adv. Boundary Element Methods, San Antonio, Texas, (1987), 215-222.

[22] Kunihiro, N., Hayami, K. and Sugihara, M., Automatic numerical integration of nearly singular boundary element integrals, in T. Ushijima, Z. Shi and T. Kako eds., Adv. Numer. Math., Proc. 2nd Japan-China Seminar on Numerical Mathematics, Tokyo, 1994, Lecture Notes in Numer. Appl. Anal., 14 (1995), 249-252.

[23] - Automatic numerical integration of nearly singular integrals in the boundary element method, in S. N. Atluri, G. Yagawa and T. A. Cruse, eds., Computational Mechanics '95, Proc. Int. Conf. on Computational Engineering Science, Hawaii, SpringerVerlag, 2 (1995), 2841-2846. 
[24] Kunihiro, N., Hayami, K. and Sugihara, M., Automatic numerical integration for the boundary element method using variable transformation and its error analysis, Trans. Japan Soc. Ind. Appl. Math., 5 (1995), 101-119, (in Japanese).

[25] Lachat, J. C. and Watson, J. O., Effective numerical treatment of boundary integral equations: A formulation for three-dimensional elastostatics, Int. J. Numer. Methods Eng., 10 (1976), 991-1005.

[26] McNamee, J., Error-bounds for the evaluation of integrals by the Euler-Maclaurin formula and by Gauss-type formulae, Math. Comp., 18 (1964), 368-381.

[27] Schwab, C., Variable order composite quadrature of singular and nearly singular integrals, Computing, 53 (1994), 173-194.

[28] Sládek, V. and Sládek, J., Non-singular boundary integral representation of stresses, Int. J. Numer. Methods Eng., 33 (1992), 1481-1499.

[29] Telles, J. C. F., A self-adaptive coordinate transformation for efficient numerical evaluations of general boundary element integrals, Int. J. Numer. Methods Eng., 24 (1987), 959-973.

[30] Takahasi, H. and Mori, M., Error estimation in the numerical integration of analytical functions, Report of the Computer Centre University of Tokyo, 3 (1970), 41-108.

[31] Double exponential formulas for numerical integration, Publ. RIMS, Kyoto Univ., 9 (1974), 721-741. 